\title{
Some Theorems in Group Velocity
}

\author{
By P. M. Davidson
}

(Received 15th February, 1951.)

1. Introduction. We consider a medium in which the equation satisfied by a disturbance $\phi(x, t)$ is capable of solutions of the form $\sin (\nu x-\omega t)$, with $\nu$ and $\omega$ real but not necessarily of the same sign. If the phase velocity $U=\omega / \nu$ is not of constant magnitude the medium is said to be dispersive, and the group velocity $V$ may conveniently be defined as $d \omega / d \nu$, an expression easily re-written in other familiar forms. From this definition the two physical interpretations of $V$ may easily be seen. In one interpretation we consider a superposition of two harmonic waves with slightly different $\nu$ (and $\omega$ ); the velocity of advance of the group form, being the velocity of advance of a point at which the two waves have a constant phase difference, is $d \omega / d \nu$. From the system just described we readily obtain the alternative interpretation of $V$ as a velocity of propagation of "energy", which for this purpose could be defined as any quantity whose spatial density $\epsilon$ satisfied a relation ${ }^{1}$ of the form $\dot{\epsilon}=-S^{\prime}$, where $\epsilon$ and $S$ are functions (of the quantities specifying the disturbance, and of their various differentials) such that $\epsilon$ and $S$ are zero in the undisturbed medium and $\epsilon$ has a non-zero mean value in a harmonic wave. For then, if we consider for example a region $P P_{1}$ of space, of length $L$, in which, at a certain time, the group pattern of the double wave ranges from its very small minimum to its maximum, of amplitude say $A$, at $P_{1}$, then, from the change in the energy content of this space when the group pattern moves by a small fraction of $L$, we see at once that if $\bar{\epsilon}$ is the mean energy density in the region of $P_{1}$ the mean value of $S$ in the plane $P_{1}$ is $(d \omega / d \nu) \bar{\epsilon}$; that is, $V \bar{\epsilon}$ is the mean forward flow of energy per unit area per unit time in a region where the disturbance is $A \sin (\nu x-\omega t)$ with mean energy density $\bar{\epsilon}$. It will be noted that in this interpretation of $V$ the idea of the double wave is no longer present.

For subsequent use it may be mentioned that a third physical interpretation of $V$ is known, though as it stands it is of a rather academic

1 The point and dash mean $\partial / \partial t$ and $\partial / \partial x$; and the range of all the integrals in the paper is from $-\infty$ to $\infty$, except where otherwise obvious. 
nature, since it is concerned with a state attained asymptotically at very large time. It may be described roughly by saying that if we have initially a disturbance in a finite region round the origin, the wave lengths present in a Fourier analysis of it will, after a very long time $t$, have separated out into a spectrum in space, the disturbance in the region of a point distant $V(\lambda) t$ from the origin being then a harmonic wave of length $\lambda$ and of amplitude indicated by the original Fourier analysis. If, for example, in a dispersive medium of infinite extent, a real quantity $\phi$ (falling off sufficiently rapidly at large $|x|$ at $t=0$ ) can be represented at $t>0$ by

$$
\phi=(2 \pi)^{-1 / 2} \int F(\nu) \exp \{i(\nu x-\omega t)\} d \nu
$$

where $\omega$ has the same sign as $\nu$, and $|\omega|$ is a function of $|\nu|$, and if there are no two $|\nu| \mathrm{s}$ with the same $V$, then at sufficiently large $t$ the disturbance $\phi$ in the region of $x=V t$ is a harmonic wave of length $2 \pi /|\nu|$, phase velocity $\omega / \nu$, and amplitude $A(x, t)$ conveniently specified by saying that the relation $\int \phi^{2} d x=2|F(|\nu|)|^{2} d|\nu|$ (which when integrated with respect to $|\nu|$ holds at all times) ultimately holds for each element $d|\nu|$, if the integral on the left is taken over the corresponding range, which is of length $\Delta x=t . d V$; the integral is evidently $\frac{1}{2} A^{2} \Delta x$. The statement is readily generalised for other cases such as those in which $\phi$ is a complex quantity, or cannot be represented by the single integral (1).

2. The theorems which will be established in the present paper exhibit a different aspect of group velocity. It will be shown that in various types of dispersive media, of infinite extent, the motion resulting from an arbitrary initial state of disturbance is characterised by certain quantities which are independent of time and may be expressed as suitable averages of the group velocity (or a power of it) over the range of waves present in a Fourier analysis of the original disturbance.

As a first example let us suppose that at $t>0$ a quantity $\phi$, in general complex, is given by

where

$$
\phi=(2 \pi)^{-1 / 2} \int f(\nu, t) \exp i \nu x d \nu
$$

$$
f(\nu, t)=F(\nu) \exp -i \omega t
$$

and $\omega$ is a function of $\nu$, say $h(\nu)$. It will be assumed that $\phi$, which by (2) may be described as the Fourier transform of $f$, satisfies the restrictions 
needed to justify the following operations. By applying the Fourier inversion theorem to (2) and then differentiating $m$ times with respect to $\nu$ we see that $x^{m} \phi$ is the transform of $i^{m} \partial^{m} f / \partial \nu^{m}$. By (3), $\partial^{m} f / \partial \nu^{m}$ is $\exp (-i \omega t)$ multiplied by a power series in $t$ in which the term of highest degree is $(-i t V)^{m} F$. Differentiating (2) $n$ times with respect to $x$, we see that $\partial^{n} \phi / \partial x^{n}$ is the transform of $(i \nu)^{n} f$. Using these results we are able, by Parseval's formula, to write $\int x^{m} \phi\left(\partial^{n} \phi^{*} / \partial x^{n}\right) d x$ as an integral (with respect to $\nu$ ) of which the $m$-th time differential can be immediately written down. We find, in fact, that if (using a star to mean a harmonic conjugate) $\epsilon_{n}$ is defined by

$$
\epsilon_{n}=\frac{A^{n}}{2}\left\{i^{n} \phi \frac{\partial^{n} \phi^{*}}{\partial x^{n}}+i^{-n} \phi^{*} \frac{\partial^{n} \phi}{\partial x^{n}}\right\}
$$

and $X_{n}^{m}$ by $\int x^{m} \epsilon_{n} d x / \mathrm{E}_{n}$, where $\mathrm{E}_{n}=\int \epsilon_{n} d x$

then if we write

$$
d \mathrm{E}_{n}=(A v)^{n} F F^{*} d \nu
$$

we have

$$
\mathbf{E}_{n}=\int d \mathbf{E}_{n}
$$

and

$$
\left\{d^{m} X_{n}^{m} / d t^{m}\right\} / m !=\int V^{m} d \mathrm{E}_{n} / \mathrm{E}_{n}
$$

It is evident that the expression (4), and hence also (5), is independent of time. (In particular cases the denominator in this last expression may be zero, or the numerator or denominator may be a divergent integral; in such cases the expression has, of course, no meaning.) It will be noted that the theorem still holds if, throughout, $\phi$ is replaced by, say, $\dot{\phi}$ or $\phi^{\prime}$, since these quantities are also of the form (2).

If the equation of motion of the medium is

$$
\dot{\phi}=\Sigma i^{-(p+1)} a_{p} \partial^{p} \phi / \partial x^{p}
$$

(where $a$ 's are real and $h(v)$ is evidently $\Sigma a_{p} \nu^{p}$ ), a value $\phi(x)$ specified for $\phi$ at $t=0$ evidently determines $\phi(x, t)$ at all later times. Hence (2), with this $h(\nu)$, being a solution of the equation of motion, is equal to this $\phi(x, t)$ if at $t=0$ it is equal to $\phi(x)$; and this it will be if in (3) we insert the $F$ calculated from $\phi(x)$ by the Fourier reciprocal theorem. In the case 
of the one-dimensional Schrödinger equation, which may be written $\dot{\phi}=i a \phi^{\prime \prime}$ and is thus a particular case of $(6), \phi$, if normalised so as to give $\mathrm{E}_{0}$ a value $M$, is interpreted as representing a large number of equal point-masses of total mass $M$ in free motion parallel to the $x$-axis. If $A$ is given the value $2 a$, then $\epsilon_{0}, \epsilon_{1}, \epsilon_{2}$ are the expressions which are interpreted as the amounts, per unit length, of mass, momentum and vis viva. The expression $f f^{*} d \nu$ is interpreted as the mass of particles which have velocities $d \omega / d \nu$ in the corresponding small range. Thus in the case of the Schrödinger equation, (5) is the wave mechanical transcription of the kinematical theorem, applicable to the corresponding physical system

$$
\left\{d^{m} X_{n}{ }^{m} / d t^{m}\right\} / m !=\left(\Sigma v^{m+n}\right) /\left(\Sigma v^{n}\right)
$$

independent of time.

3. For many of the wave motions occurring in physics the differential equation of motion is not such that a single scalar function of $x$, specified for $t=0$, determines the subsequent motion. More often the equation is such that the motion is determined by the values at time zero of two real scalar quantities (such as a real scalar and its time differential coefficient); and the expressions for such quantities, in the infinite medium, are of the form

where $\quad f_{-}=F_{-} \exp -i \omega t ; f_{+}=F_{+} \exp i \omega t$ and $|\omega|$ is a function of $|\nu|$, say $h(|\nu|)$.

$$
\left.\begin{array}{c}
\phi=(2 \pi)^{-1 / 2} \int\left(f_{-}+f_{+}\right) \exp i \nu x d \nu \\
f_{-}=F_{-} \exp -i \omega t ; f_{+}=F_{+} \exp i \omega t
\end{array}\right\}
$$

$F_{-}$and $F_{+}$are readily calculated from the specified initial conditions by the inversion formula.

If either $F_{-}$or $F_{+}$is zero, so that only the + or - waves are present, it is evident that the arguments leading to the theorem (5) are still valid; whether the motion expressed by the general solution (7) is characterised by any such constants is a question that will now be considered.

In that general case it will be found from (7) by using Parseval's formula that if $a$ and $A$ are constants

$$
\begin{aligned}
& I=\frac{a}{2} \int\left(\dot{\phi}^{2}-\dot{\phi}\right) d x \text { is independent of time } \\
& J=-A \int \dot{\phi} \phi^{\prime} d x \quad \text { is independent of time. }
\end{aligned}
$$

These two results ${ }^{1}$ are useful for the purpose.

\footnotetext{
1 It will be found that there are also a large number of such expressions containing higher differential coefficients.
} 
As an example we may consider an irrotational disturbance of small amplitude in an inviscid incompressible fluid of finite (or infinite) depth $H$, acted on by gravity. The typical harmonic solution and the function $h(|\nu|)$ are well known. We will take the $y$-axis as directed upwards, with $y$ zero at the surface of the undisturbed fluid. In the expressions of type (7) for quantities which vary with $y, F_{-}$and $F_{+}$are functions of $v$ and $y$, but this does not affect the derivation, from (7), of (8) and (9), which are thus true at every $y$. The general motion considered may be thought of as having been established by applying forces to the upper surface of the fluid prior to $t=0$. The vertical surface-displacement and velocity, say $\eta(x)$ and $\dot{\eta}(x)$ at $t=0$, determine the subsequent motion, the $F_{-}$and $F_{+}$ in expressions of the type (7) being readily calculated from them. If now we consider a unit thickness in the $z$ direction, and if the energy $\epsilon$ in a vertical column, of unit length in the $x$ direction and extending from the bottom to the surface, is defined as the sum of the kinetic energy in the whole column and the work obtainable by redistributing the surface column of height $\eta$ as a thin sheet at the level of the undisturbed surface (with an analogous definition where $\eta$ is negative), then if $\phi$ is the velocity potential and if $a$ and $A$ are given the value $\rho / g$, the constant integral $I$, taken at $y=0$, is equal to the total energy, say $\mathrm{E}$ (a formula which is also true for one wave-length of a harmonic wave if present alone). Moreover $\dot{\epsilon}=-S^{\prime}$, where $\int S d x=g \int_{-H}^{0} J d y$, and, since (9) holds at every $y$, we see that $\int S d x$ is independent of time, and hence (integrating $x \dot{\epsilon} d x$ by parts) that so also is $(d / d t) \int x \epsilon d x / \mathrm{E}$, the velocity $\dot{X}$ of the centre of energy ${ }^{1}$. For evaluating these constant quantities we may evidently choose a time so great that the frequency distribution has become a spatial distribution, as mentioned in $\S 1$. Thus if the functions $F_{-}$and $F_{+}$are those in the expression of type (7) for $g \eta$ (which in this motion is the value of $\dot{\phi}$ at $y=0$ ) and if we write $d \mathrm{E}_{-}=a F_{-} F_{-} * d \nu$ and $d \mathrm{E}_{+}=a F_{+} F_{+} * d \nu$ we have

$$
\mathrm{E}=\int\left(d \mathrm{E}_{-}+d \mathrm{E}_{+}\right) \text {and } \dot{X}=\int\left(V_{-} d \mathrm{E}_{-}+V_{+} d \mathrm{E}_{+}\right) / \mathrm{E}
$$

1 Similarly, of course, in media where there are relations of the form $\dot{\epsilon}=-S^{\prime}, \dot{S}=-R^{\prime}$, and $\int \dot{R} d x=0$, the quantity $d^{2}\left\{\int x^{2} \in d x\right\} / d t^{2}$ is independent of time, and the expression for it as $\mathbf{E}$ multiplied by an average of $V^{2}$ is easily writton down. It is also evident that, owing to the ultimate sorting out of the frequencies in space, there will be a large number of $X^{m}$ 's whose $m$-th time differential coefficients, though not constant, will tend with increasing time to constant values given by simple expressions, 
In visualising this average of $V$, it should be remembered that at a given wave-length its values $V_{-}$and $V_{+}$in the - and + waves are, by our definitions, equal and opposite.

Returning to the general class of wave motions satisfying (7) we see that another quantity, constant during the motion and having the dimensions of a velocity, may be obtained by dividing (8) by (9). Writing

$$
d J_{-}=A F_{-} F_{-} * d v / U_{-} \text {and } d J_{+}=A F_{+} F_{+} * d v / U_{+}
$$

where the $F$ 's are those of $\dot{\phi}$, we have

$$
J=\int\left(d J_{-}+d J_{+}\right) \text {and } I / J=\frac{a}{A} \int\left(U_{-} d J_{-}+U_{+} d J_{+}\right) / J .
$$

Thus, putting $a=A$, we can regard $I / J$ as an average not of the group but of the phase velocity; but this is a rather artificial way of regarding it. It is of quite a different nature from such a quantity as $\dot{X}$ and cannot be thought of as the velocity of a moving point having a simple physical significance. Its meaning may be exemplified by taking the case of the fluid waves and considering the two integrals $I$ and $J$ at $y=0$. If we put $a=A=\rho / g, I$ is, as remarked above, the total energy : and (since $\dot{\phi}$ is $g \eta$ ) $J$ is the total momentum, in the $x$ direction, in the columns of height $\eta$ in the regions where $\eta$ is positive, together with an analogous contribution from the regions where $\eta$ is negative; it is also equal to the momentum in the $x$ direction generated in the fluid by the forces acting on the upper surface during the original setting up of the motion. Thus the expression for $I / J$ asserts that the total energy is obtained by multiplying each of the elements $d J_{-}$and $d J_{+}$of momentum by the corresponding phase velocity and summing them.

In conclusion it should be mentioned that there are complex dispersive media in which four quantities can be assigned arbitrary initial values: as would be expected the general solution corresponding to (7) now consists of four integrals. They are made possible by there being, in such media, two possible frequencies for each wave-length.

\section{University College,} Swansea. 